\title{
Philosophiques
}

\section{Deux sources de la moralité}

\section{Philip Pettit}

Volume 28, numéro 1, printemps 2001

\section{La nature des normes}

URI : https://id.erudit.org/iderudit/004967ar

DOI : https://doi.org/10.7202/004967ar

Aller au sommaire du numéro

\section{Éditeur(s)}

Société de philosophie du Québec

\section{ISSN}

0316-2923 (imprimé)

1492-1391 (numérique)

Découvrir la revue

\section{Citer cet article}

Pettit, P. (2001). Deux sources de la moralité. Philosophiques, 28(1), 173-203. https://doi.org/10.7202/004967ar

\section{Résumé de l'article}

Comment chercher à situer, dans l'expérience humaine, les termes ou les concepts moraux? Autrement dit, où, dans l'expérience, la morale devient-elle saillante pour nous ? C'est par le biais d'une généalogie naturaliste qu'il nous faut envisager la problématique, dans la mesure où nous ne possédons pas un sens moral irréductible par lequel des propriétés morales irréductibles nous seraient connues. Je soutiens que si des sujets intentionnels n'ont nul besoin de disposer de concepts normatifs, il en sera autrement pour des créatures discursives. Ces dernières, afin de raisonner ensemble, devront avoir accès aux concepts normatifs inférentiels et c'est par deux voies distinctes qu'elles seront amenées à prendre note des considérations morales. La première engage les individus qui privilégient le discours, comme forme d'interaction; la seconde, ceux qui étendent cette pratique discursive au domaine du sentiment. Le privilège accordé au discours et l'exercice discursif du sentiment constituent les deux sources distinctes d'une conceptualisation morale, évoquées dans le titre de cet article. Dans une brève conclusion, il est suggéré que les différents concepts qu'elles fournissent représentent des candidats attractifs rivaux pour la construction de la théorie morale.
Ce document est protégé par la loi sur le droit d'auteur. L'utilisation des services d’Érudit (y compris la reproduction) est assujettie à sa politique d'utilisation que vous pouvez consulter en ligne.

https://apropos.erudit.org/fr/usagers/politique-dutilisation/ 


\title{
Deux sources de la moralité
}

\author{
PHILIP PETTIT \\ Australian National University \\ pnp@coombs.anu.edu.as
}

\begin{abstract}
RÉSUMÉ. - Comment chercher à situer, dans l'expérience humaine, les termes ou les concepts moraux? Autrement dit, où, dans l'expérience, la morale devient-elle saillante pour nous ? C'est par le biais d'une généalogie naturaliste qu'il nous faut envisager la problématique, dans la mesure où nous ne possédons pas un sens moral irréductible par lequel des propriétés morales irréductibles nous seraient connues. Je soutiens que si des sujets intentionnels n'ont nul besoin de disposer de concepts normatifs, il en sera autrement pour des créatures discursives. Ces dernières, afin de raisonner ensemble, devront avoir accès aux concepts normatifs inférentiels et c'est par deux voies distinctes qu'elles seront amenées à prendre note des considérations morales. La première engage les individus qui privilégient le discours, comme forme d'interaction; la seconde, ceux qui étendent cette pratique discursive au domaine du sentiment. Le privilège accordé au discours et l'exercice discursif du sentiment constituent les deux sources distinctes d'une conceptualisation morale, évoquées dans le titre de cet article. Dans une brève conclusion, il est suggéré que les différents concepts qu'elles fournissent représentent des candidats attractifs rivaux pour la construction de la théorie morale.
\end{abstract}

ABSTRACT. - Where in human experience are moral terms or concepts grounded; where in experience does the moral becomes salient to us? The question calls for a naturalistic genealogy, so far as we are not possessed of an irreducibly moral sense whereby irreducibly moral properties might be revealed to us. I argue that intentional subjects need not have any normative concepts whatsoever but that creatures who are discursive do. They will have to access inferentially normative concepts so far as they reason together and there are two separate ways in which they will be disposed to register moral considerations. The first involves them privileging discourse as a form of interaction and the second involves them extending such discourse to the realm of sentiment. Privileging discourse and discursifying sentiment are the two distinct sources of moral conceptualisation that are signaled in the title of the paper, and a brief conclusion suggests that the different concepts they provide represent rival attractors in the construction of moral theory.

C'est sur une question propre à l'épistémologie de l'éthique ou de la moralité que cet article trouve son point de départ. Il ne s'agit pas de cette question commune, axée sur le jugement, qui vise à établir comment les jugements moraux sont confirmés et si ce mode de confirmation peut nous apporter des raisons convaincantes quant aux possibilités du discours éthique. Au contraire, la question est celle, d'ailleurs moins fréquemment formulée, qui se centre sur la notion du concept et qui cherche à situer, dans l'expérience humaine, les termes ou concepts moraux. Pour le dire autrement, où, dans l'expérience, la morale devientelle saillante pour nous ? Cette question fut centrale pour l'épistémologie, dans

PHILOSOPHIQUES 28/1 — Printemps 2001, p. 173-203 


\section{4 · Philosophiques / Printemps 2001}

la forme qu'elle a prise chez des penseurs tels que Locke, Hume et Kant, et demeure aujourd'hui de première importance ${ }^{1}$.

C'est par le biais d'une généalogie naturaliste qu'il est nécessaire d'envisager cette problématique. Je suppose en effet que nous ne possédons pas un sens moral irréductible, par lequel des propriétés morales irréductibles nous seraient révélées. J'admets plutôt que nous, êtres humains, ne sommes pas dotés de facultés non naturelles de perception et de cognition et, qu'en tous les cas, il n'y a rien de non naturel que de telles facultés puissent saisir. La question est donc de savoir ce qu'il en est de l'expérience naturaliste - d'une expérience d'un type tel qu'elle ne soulève aucune préoccupation particulière pour une conception scientifique du monde - et qui peut occasionner une conceptualisation morale et créer une opportunité pour un déploiement fécond des termes moraux.

L'analyse que l'on propose des termes ou concepts moraux attirera souvent l'attention vers une généalogie entendue en ce sens. Si l'on présente une analyse non cognitiviste de l'évaluation morale comme étant une espèce d'expression ou de projection d'attitudes, par exemple, cela mènera alors à une autre interprétation de ce qui, dans l'expérience, occasionne une telle évaluation. ${ }^{2}$. Pourtant, l'analyse des termes moraux laissera souvent la question généalogique en suspens. Supposons que nous postulions une analyse en réseau des termes moraux selon laquelle les significations de ces différents termes sont établies grâce aux liens que nous reconnaissons parmi ces termes et entre ces derniers et des termes de type non moral ; je suis moi-même en faveur d'une approche de ce type ${ }^{3}$. Une telle analyse en réseau présuppose que ces concepts, formant des réseaux, ont, à certains endroits, des points de contact avec l'expérience ; certains concepts moraux - certains des termes impliqués dans la toile du discours moral — doivent avoir un ancrage dans

1. Pour une discussion récente et de grande importance sur le problème de l'origine des concepts, voir Korsgaard, C. M., The Sources of Normativity, New York, Cambridge University Press ; voir aussi Korsgaard, C. M., "Self-Constitution in the Ethics of Plato and Kant ", The Journal of Ethics, 3, 1999, p. 1-29. Mon approche se distingue de la sienne, dans la mesure où c'est plutôt à un niveau social que réflexif qu'elle fait remonter l'origine de la conceptualisation éthique ; à cet égard, elle se rapproche davantage de celle proposée par Postma, G. M., "Morality in the First Person Plural ", Law and Philosophy, 14, 1995, p. 14-34. Pour une approche qui soutient une thèse non cognitiviste cette fois, mais dans laquelle un grand nombre des thèmes impliqués restent favorables à cette problématique, voir Gibbard, A., Wise Choices, Apt Feelings, Oxford, Oxford University Press, 1990.

2. Pour un aperçu de l'approche cognitiviste que je défends, voir Jackson, F. et P. Pettit, "Moral Functionalism and Moral Motivation ", Philosophical Quarterly, 45, 1995, p. 20-40; et Pettit, P., "Embracing Objectivity in Ethics ", dans Leiter, B., dir., Objectivity in Law and Morals, Cambridge, Cambridge University Press, 2000. Pour une critique de l'expressivisme et une argumentation indirecte en faveur du cognitivisme, voir Jackson, F. et P. Pettit, " A Question for Expressivists », Analysis, 58, 1998, p. 239-251.

3. Hurley, S., Natural Reasons, New York, Oxford University Press ; Jackson, F. et P. Pettit, "Moral Functionalism and Moral Motivation", p. 20-40; Wedgwood, R., «Conceptual Role Semantics for Moral Terms », Philosophical Review, à paraître. 
l'expérience, ne serait-ce que de manière provisoire, par des moyens plus directs d'ordre sémantique. Quoique l'analyse reconnaîtra la nécessité de ces points de contact, elle n'aura peut-être que peu à dire sur la manière dont ils sont établis ; elle risque bien de laisser la question généalogique sans réponse.

Bien que je favorise une analyse cognitiviste ou en réseau des termes moraux, je tente ici d'étudier la question généalogique en faisant abstraction des problèmes propres à l'analyse. La façon dont je discute présuppose souvent que les concepts moraux ont pour fonction d'attribuer des propriétés afin que, comme le soutiennent les cognitivistes, les évaluations dans lesquelles ils figurent soient vraies ou fausses. Mais cette thèse cognitiviste, je ne la présuppose qu'aux endroits où ceux qui estiment que ces évaluations sont simplement des expressions d'une attitude non cognitive seront capables de remanier l'argumentation de façon à ce qu'elle soit favorable à leur vision des choses. Considérons, par exemple, l'exposé relatif à la manière dont les sentiments donnent lieu à une conceptualisation morale et qui se trouve introduit dans la dernière section de cet article. Si l'exposé s'établit en des termes favorables à la thèse cognitiviste, il ne devrait pas être difficile de voir comment s'articulerait l'équivalent d'une explication non cognitivisite.

Ma discussion est structurée en cinq sections. Dans la première, je soutiens que des sujets intentionnels - des sujets qui forment des croyances et des désirs, et agissent sur cette base - n'ont nul besoin de disposer d'aucun type de concepts normatifs. Dans la seconde, je soutiens que des créatures qui sont discursives aussi bien qu'intentionnelles, qui expriment leurs états intentionnels au moyen de signes volontaires communs, doivent posséder certains concepts normatifs, c'est-à-dire ceux associés au raisonnement. Dans la troisième section, je fais remarquer que ces concepts normatifs nécessaires au discours ne sont pas des concepts moraux proprement dits. Finalement, j'examine, dans les deux dernières sections deux voies par lesquelles les considérations morales peuvent devenir saillantes pour des créatures discursives. La première concerne les individus qui privilégient le discours comme forme d'interaction; la seconde concerne ces mêmes individus lorsqu'ils étendent ce discours au domaine du sentiment. C'est le privilège accordé au discours et l'exercice discursif du sentiment qui constituent les deux sources distinctes de la conceptualisation morale, sources évoquées dans le titre de cet article. Je soutiens, dans une brève conclusion, que les différents concepts qu'ils fournissent représentent des candidats attractifs rivaux pour la construction de la théorie morale.

\section{Les sujets intentionnels peuvent être dépourvus de concepts normatifs}

Les êtres humains sont des créatures intentionnelles qui forment des croyances et des désirs et qui agissent sur cette base, d'une façon plus ou moins rationnelle. L'expérience des créatures intentionnelles peut-elle, de manière 
certaine, rendre les considérations morales saillantes et, par conséquent, offrir une circonstance propice à la formation des concepts correspondants ? Non, elle ne le peut pas. Dans cette section, je soutiens qu'il est possible, au contraire, d'être un sujet intentionnel tout en ne disposant d'aucun type de concepts moraux.

Tout sujet intentionnel doit être conçu de telle sorte qu'il se comporte adéquatement, sous certains aspects représentationnels et connexes, et être un sujet intentionnel ne signifie rien de plus et rien de moins que de satisfaire à de telles spécifications ${ }^{4}$. C'est en tous les cas ce que je supposerai. Le sujet intentionnel se représentera les choses telles qu'elles apparaissent à l'intérieur des limites de son organisation perceptuelle et cognitive, actualisant ses représentations de manière appropriée à la lumière de nouveaux inputs. De plus, il agira selon ce qui favorise ses désirs - désirs qui, selon toute vraisemblance, reflètent ses buts et besoins globaux - à la lumière de ses représentations ou croyances. Évidemment, il peut bien ne pas faire montre d'un comportement parfaitement adéquat au niveau pratique ou au niveau cognitif mais il devra bien performer - et cela, peut-être, à la suite d'une certaine histoire ou d'une certaine organisation - dans le cadre de ce qui est considéré comme des limites de faisabilité et des circonstances favorables.

Les succès rationnels dont le sujet intentionnel doit faire preuve, selon cette analyse, sont tout à fait impressionnants. Or, il s'avère toutefois que même un système mécanique ou organique relativement simple peut être intentionnel en ce sens. Sans avoir lui-même aucun type de contrôle sur le processus, un animal ou un robot pourrait être ainsi constitué qu'il parvienne à s'ajuster à l'entrée d'informations d'une façon relativement exacte, formant des croyances qui déterminent, en présence d'un certain état ou de désirs situationnels variables, ce que fait le système. Ses croyances pourraient être mises à jour à la lumière d'un input perceptuel, suivant la routine d'un modèle correctement programmé, et elles pourraient se combiner aux désirs de la créature en question en suivant une routine similaire dans cette actionci ou dans celle-là. À tout le moins devraient-elles parvenir à ce résultat à l'intérieur de limites intuitives de faisabilité et sous des circonstances intuitivement favorables.

Les croyances et les désirs d'un sujet simple comme celui-ci seront liés à des contraintes ou à des normes, pour autant qu'il leur faille se conformer au modèle précédemment décrit. Les croyances rencontreront certaines conditions d'entrée et de sortie : par exemple, elles ont une tendance à entrer en

4. Sur la notion des spécifications au niveau du plan, voir Dennett, D., The Intentional Stance, Cambridge (Mass.), MIT Press, 1987 ; Pettit, P., The Common Mind: An Essay on Psychology, Society and Politics, New York, Oxford University Press, 1993, édition de poche 1996, chap. 1 ; et Railton, P., " On the Hypothetical and Non-Hypothetical in Reasoning about Belief and Action », dans Cullity, G. et B. Gaut, dir., Ethics and Practical Reason, Oxford, Oxford University Press, 1997. 
scène là où le sujet trouve de bonnes raisons, et à sortir là où il trouve des raisons opposées. Et aussi bien les désirs que les croyances du sujet rencontreront un éventail de conditions de performance. Ainsi, le désir que p aura tendance à interagir avec la croyance que si $\mathrm{p}$ alors $\mathrm{q}$, pour générer le désir que $\mathrm{q}$ ou affaiblir le désir que $\mathrm{p}$. La croyance que $\mathrm{p}$ aura tendance à interagir avec la croyance que si $\mathrm{p}$ alors $\mathrm{q}$, pour générer la croyance que $\mathrm{q}$ ou affaiblir l'une des autres croyances. Le désir que $\mathrm{p}$ aura tendance à interagir avec la croyance que l'on peut faire en sorte que $\mathrm{p}$ en faisant $\mathrm{X}$, afin de générer l'action de faire X. Et ainsi de suite.

Même si des sujets simplement intentionnels devront satisfaire à de telles contraintes ou normes de rationalité - plus précisément, à celles de la rationalité inférentielle - ils n'auront pas, ce faisant, conscience des exigences impliquées par ces normes. En formant une nouvelle croyance selon ce que dicte la perception, ils ne prendront pas note que la perception fait de cette croyance celle qu'il est juste de soutenir. En formant un désir selon ce que dictent les amendements apportés à leurs croyances ou selon une inclination spontanée, ils ne prendront pas note du fait que ce sont ces croyances ou ce but auquel ils sont enclins spontanément qui font que ce désir est plus ou moins approprié. En s'engageant vers l'action sur la base de leurs croyances et désirs, ils ne prendront pas note que cette action est la chose à faire en raison des fins qui les attirent et des opportunités disponibles. Ces notions normatives au sujet de ce qu'il est juste de croire, de ce qu'il est approprié de désirer ou de ce qui doit être fait n'auront pas de place dans leur psychologie. Ces sujets se conformeront peut-être aux exigences des normes pertinentes, mais ils ne pourront les conceptualiser comme des exigences auxquelles ils doivent se conformer.

En conséquence, pour les créatures envisagées, percevoir les choses sous un certain angle reviendra précisément à croire qu'elles sont ainsi; de même, être enclin à faire que les choses soient d'une certaine manière reviendra précisément à agir, lorsque c'est possible, pour faire en sorte qu'elles soient ainsi, c'est-à-dire ni plus ni moins que de former un désir non conditionnel ou une disposition à agir de cette manière. Par contre, pour des sujets tels que nous, êtres humains, percevoir les choses sous un certain angle avoir une perception faillible de ces choses - nous donnera une raison inférentielle de croire qu'elles sont ainsi, mais une raison que nous pouvons remettre en cause. De plus, pour des sujets tels que nous, être enclins à faire que les choses soient d'une certaine manière nous donnera une raison inférentielle de faire en sorte qu'elles soient ainsi, mais une raison que nous pouvons ignorer ou inhiber. Pour nous, la perception et l'inclination existent comme des états que nous pouvons conceptualiser en tant que tels, ce qui leur donne une autorité présomptive mais pas nécessairement conclusive dans la détermination de nos croyances finales et de nos désirs non conditionnels. Mais du point de vue des créatures simplement intentionnelles que nous avons imaginées, la perception et l'inclination n'auront ni une telle visibilité ni un tel rôle. 
C'est de manière invisible et inéluctable que la perception et l'inclination seront en opération lorsqu'elles détermineront, chez de tels sujets, comment le monde se présente à eux et comment il peut susciter une action de leur part. Ces créatures mues par certaines perceptions et inclinations ne sauront considérer ces états pour ce qu'ils sont pour nous : des indicateurs strictement présomptifs et non contraignants de ce qui est le cas et de ce que l'on doit faire. Elles vont examiner leurs perceptions et considérer le monde tel qu'il se présente, selon les croyances qu'elles forment d'une manière non réfléchie ; elles vont examiner leurs inclinations et considérer le monde tel qu'il les interpelle à l'action, selon les désirs qu'elles conçoivent d'une manière non réfléchie. De tels sujets seront les spectateurs captifs de leurs représentations perceptuelles et les exécutants captifs des fins qu'ils sont enclins à poursuivre. Ils seront les esclaves de la perception et de l'inclination.

Le résultat de tout ceci est qu'il est difficile d'expliquer la façon dont la morale devient saillante pour des créatures telles que nous seulement à partir du fait que nous sommes des sujets intentionnels. Rien dans l'intentionnalité ellemême ne peut expliquer pourquoi des sujets intentionnels voient les choses d'une manière qui puisse donner lieu à l'introduction des termes et des concepts normatifs. Si nous voulons offrir une généalogie naturaliste de ces termes et concepts, il nous faudra donc partir d'une image plus riche des êtres humains.

\section{Des sujets discursifs disposeront de concepts normatifs inférentiels}

\subsection{De l'intentionnalité au discours}

Nous, êtres humains, ne sommes pas que de simples sujets intentionnels qui forment des croyances et des désirs et agissent sur cette base, d'une manière plus ou moins rationnelle. L'une des caractéristiques les plus remarquables de notre espèce est que nous sommes aussi des créatures conversationnelles ou discursives ${ }^{5}$. Nous ne formons pas seulement des croyances et des désirs tout comme s'en montrent capables de nombreux animaux non-humains, selon la théorie esquissée dans la dernière section. Nous pouvons aussi exprimer de manière volontaire ou intentionnelle comment, à la lumière de nos croyances et de nos désirs, les choses se présentent. Nous n'avons pas que cette capacité stricte à croire que $\mathrm{p}$, nous pouvons affirmer que $\mathrm{p}$; nous pouvons utiliser un signe volontaire, selon l'expression de Locke, afin de représenter comment les choses se présentent, étant donné cette croyance ${ }^{6}$. Nous n'avons pas seulement le désir que q, nous pouvons affirmer que la possibilité que q est attrac-

5. Deacon, T., The Symbolic Species : The Co-Evolution of Language and the Human Brain, London, Penguin, 1997.

6. Locke, John, An Essay Concerning Human Understanding, Nidditch, P. H., dir., Oxford, Oxford University Press, 1975, livre III, chap. 2 (Essai philosophique concernant l'entendement humain, trad. Coste, Paris, Vrin, 1972). 
tive, ou que sais-je encore ; nous pouvons utiliser un signe volontaire pour représenter comment les choses se présentent, étant donné ce désir. Nous pouvons exprimer nos croyances à l'aide de phrases régulières, spécifiant un contenu, et nous pouvons exprimer nos désirs par des phrases qui prédiquent quelque chose d'attractif ou de similaire au sujet des contenus désirés.

Que nous soyons des créatures discursives signifie que nous avons accès à des signes volontaires de cette sorte et que nous les employons dans le but d'arriver, par une voie exempte de coercition et de collusion, à une idée commune sur certains sujets. Nous utilisons ces signes volontaires non seulement pour nous intimider, nous impressionner ou nous menacer les uns les autres -évidemment, nous pouvons faire cela aussi - mais pour discuter ou raisonner ensemble à propos de certaines questions théoriques et pratiques. Notre utilisation des signes en fonction d'un objectif de ce genre est intimement associée à notre nature d'être humain. D'ailleurs, selon une conception traditionnelle, l'utilisation discursive des signes serait nécessaire, de manière constitutive, à la capacité de penser chez les êtres humains ${ }^{7}$.

En vue de pratiquer le discours, il nous faut avoir accès au concept de signe ainsi qu'aux concepts associés que sont la signification et la représentation. Si nous utilisons les signes en tant que signes, et cela d'une manière intentionnelle ou volontaire - c'est-à-dire, selon un désir de représenter véridiquement ou non comment sont les choses, en considérant que ce sont les signes utilisés qui réalisent cette fonction représentationnelle - alors il nous faudra pouvoir concevoir les signes comme possédant une signification. Je ne tenterai pas ici d'expliquer comment des créatures naturelles peuvent en arriver à disposer du concept de signification exigé : il s'agirait, en effet, de résoudre le problème de ce que c'est que de suivre une règle ${ }^{8}$. Je présuppose que ce concept est accessible aux créatures de notre espèce - de notre espèce naturaliste - et j'essaie, à partir de cette hypothèse, de développer une explication de la façon dont nous arrivons à penser dans des termes moraux. Cela signifie que, d'un point de vue naturaliste, une dette importante reste non acquittée dans cet article.

Sans expliquer comment nous accédons au concept de signification en tant que tel, il sera cependant utile d'expliquer un tant soit peu comment nous parvenons, en tant que créatures discursives, à accorder aux mots et aux phrases des significations identiques et cela, d'ailleurs, à tel point que nous savons que nous voulons dire les mêmes choses, que nous savons que

7. Pour une défense nuancée de ce type de doctrine, voir Pettit, P., The Common Mind: An Essay on Psychology, Society and Politics, Oxford, Oxford U. Press, 1993. Une élaboration plus détaillée des implications de cette approche se retrouve dans Pettit, P. et M. Smith, "Freedom in Belief and Desire ", Journal of Philosophy, 93, 1996, p. 429-449 ; et Pettit, P., A Theory of Freedom : From Psychology to Politics, Cambridge, Polity Press, 2001.

8. On peut trouver une suggestion, traitant de quelques-unes des questions liées à cette problématique dans Pettit, P., The Common Mind: An Essay on Psychology, Society and Politics. 
nous savons cela, et ainsi de suite. L'explication que j'ai à offrir sera également pertinente dans la dernière section, alors que nous discuterons de l'exercice discursif du sentiment.

Je suggère que nous arrivons à une standardisation de la signification, au moins dans le cours général des choses, en posant trois hypothèses régulatives qui servent de cadre à nos relations les uns avec les autres. Les hypothèses sont les suivantes : d'abord, le monde de l'expérience commune met à notre disposition des candidats qui déterminent les valeurs ou les significations de nos phrases et nos mots ; deuxièmement, dans toute communauté langagière, nous pensons que nos phrases et nos mots ont une signification identique, signification dont on peut présumer du caractère saillant ; et, troisièmement, nous avons chacun, habituellement, la capacité d'utiliser nos phrases et nos mots en fonction de ces significations intentionnelles. Ces hypothèses relatives à une expérience commune, une intention commune et une compétence commune peuvent être vues comme orientant une pratique telle que, si elle est couronnée de succès, nous utiliserons effectivement nos mots et nos phrases pour dire des choses équivalentes.

Pour autant que tout se déroule aisément, les hypothèses nous conduiront à nous attribuer mutuellement ces croyances et ces désirs que nous endosserions nous-mêmes si nous utilisions les mots et les phrases que d'autres emploient. Toutefois, les choses ne seront pas toujours aussi simples. Quelqu'un pourrait utiliser une phrase donnée, qui exprimerait en apparence une croyance, par exemple « $\mathrm{p}$ », dans une situation où nous disons ou dirions «non-p ». Qu'allons-nous faire dans une telle situation ?

En réalité, nous faisons une chose dont les hypothèses régulatives contribuent à faire sens. Devant un tel type de divergence, nous nous arrêtons, présumant que quelque chose de plus ou moins contingent cloche. Nous cherchons à voir si un fait quelconque peut justifier la divergence. Par exemple, l'un de nous exprime-t-il présentement un désir et non une croyance ? La phrase « $p$ » se révèle-t-elle vague à un endroit significatif ? Sans que ce ne soit apparent, la phrase serait-elle indexicale, semblable à une phrase employant des éléments linguistiques tels que « je » ou "maintenant »? L'une de ces explications simples devait-elle s'appliquer, nous saurions alors que nos trois hypothèses régulatives n'étaient pas en danger ; cela préserverait le cadre par défaut, selon lequel nos mots possèdent une signification identique. Mais si nous échouons à trouver la cause de la divergence, nous aurons à rechercher, à un niveau plus profond, une explication qui nous permettra de maintenir les hypothèses et de préserver le cadre par défaut.

Le moyen idéal, multilatéral, de préserver le cadre par défaut consisterait à mettre sur la table les considérations qui nous guident déjà dans nos différentes stratégies - ou à rechercher des considérations pertinentes supplémentaires - et ainsi être en mesure de convenir après l'incident que l'un de nous n'avait pas pleinement compris les termes, ou bien qu'il n'était pas suffisamment informé, ou encore qu'il avait été induit en erreur à cause de 
certaines limitations, obstacles ou défaillances cognitives. Nous reviendrons plus en détail sur ce point dans ce qui va suivre. Un autre moyen, un peu moins satisfaisant à certains égards, mais toujours multilatéral, de maintenir le cadre par défaut serait de parvenir à reconnaitre que nos croyances sur des sujets collatéraux sont à ce point différentes qu'il n'y a aucune possibilité réelle pratique de résoudre la question en litige, bien qu'elle reste, en principe, susceptible de recevoir une solution. Et malgré que la voie d'une résolution multilatérale du désaccord nous soit inaccessible, nous pourrions encore, chacun à notre façon, conserver le cadre par défaut, en postulant unilatéralement que l'autre est, sans le savoir, affecté par des facteurs déstabilisants et qu'ainsi, dans cette circonstance particulière ou sur ce thème particulier, il n'est pas pleinement compétent.

Que faire si nous découvrons que nous ne pouvons pas même avoir recours à ce type d'explication unilatérale de la divergence ? À ce point, et à ce point seulement, nous aurons à admettre que le cadre par défaut est mal fondé. Nous utilisons l'un ou l'autre des mots en question sous des significations différentes, ou peut-être les mots ne possèdent-ils, à proprement parler, aucune signification. Peut-être avions-nous tort de supposer qu'il existait une chose à laquelle nos mots puissent attribuer une signification? ${ }^{9}$.

La régulation que peuvent offrir nos trois hypothèses, telle que l'analyse présentée ci-dessus le laisse prévoir, ne procède pas d'une condition, à savoir celle d'un accord préalable sur ce qui constitue les obstacles, les défaillances cognitives ou les sources collatérales de désaccords, susceptibles de déstabiliser la performance discursive. De tels facteurs déstabilisants seront identifiés au cours du processus de régulation mutuelle, au fur et à mesure que les parties prenantes à cette entreprise découvrent ensemble, graduellement, des listes de facteurs qui pourront perturber, à tout endroit, la compétence discursive. Eu égard à l'entreprise poursuivie, ces facteurs que les parties se doivent d'identifier comme étant déstabilisants sont ceux qui, du fait de leur identification en tant que déstabilisants, peuvent maximiser la convergence exempte de coercition et de collusion. Le travail de collaboration que les hypothèses régulatives cautionnent peut être vu comme une procédure de recherche par laquelle les facteurs déstabilisants sont identifiés - correctement ou peut-être incorrectement - et les hypothèses elles-mêmes, justifiées ${ }^{10}$.

Ce point mérite d'être illustré. Voyons par quels moyens vous et moi pourrions nous coordonner quand nous utilisons un prédicat de couleur comme "rouge ». Présumant une expérience commune, une intention commune et une compétence commune, nous nous attendrions, en utilisant ce prédicat, à une convergence. Si une divergence s'ensuit, et si nous ne pou-

9. Voir pourtant Pettit, P., The Common Mind: An Essay on Psychology, Society and Politics, chap. 2.

10. Pour un développement plus en détail de cette approche, voir Pettit, P., «A Theory of Normal and Ideal Conditions », Philosophical Studies, 96, 1999, p. 21-44. 
vions la justifier - disons, à partir du fait que l'un de nous utilise " rouge » dans un sens indexical tel que "cela semble rouge à mes yeux" - nous devrons alors partir à la recherche d'un facteur qui pourrait perturber, d'un côté ou de l'autre, la compétence. Nous pouvons penser à ces facteurs actuellement reconnus pour déstabiliser la compétence - porter des verres teintés, employer un éclairage au sodium, être partiellement daltonien - comme étant des facteurs qui sont identifiés précisément au cours de ce type de recherche.

La pratique décrite peut être résumée à l'aide d'une procédure que chacun d'entre nous peut être considéré comme implémentant.

- Cadre par défaut : Nos mots possèdent la même signification.

- Q1: Y a-t-il convergence entre nous dans l'usage d'un mot quelconque?

- Oui : retour au cadre par défaut.

- Non : passer à la question suivante,

- Q2 : Peut-on expliquer cette divergence dans notre usage ?

- Oui : retour au cadre par défaut.

- Non : passer à la question suivante,

- Q3 : Pouvons-nous résoudre la divergence en reconnaissant tous les deux la présence de facteurs déstabilisants?

- Oui : retour au cadre par défaut.

- Non : passer à la question suivante,

- Q4: Puis-je expliquer la divergence d'autrui en supposant la présence de facteurs déstabilisants ?

- Oui : retour au cadre par défaut.

- Non : rejeter le cadre par défaut.

J'ai indiqué que les trois hypothèses régulatives guident une pratique telle que, si elle est couronnée de succès, elle nous assure que nous avons des significations communes à l'esprit. Cette idée est relativement simple. Les hypothèses dirigeront avec succès une pratique, pour autant que nous parvenions, dans des situations de désaccord, à identifier les facteurs déstabilisants dont elles prédisent la présence ; cela devra être fait, dans la plupart des cas, par la voie d'une résolution multilatérale, quoique, à certaines occasions, nous aurons à nous en tenir à une explication unilatérale pour rendre compte des défaillances d'autrui. Si par ce biais les hypothèses connaissent un succès, la pratique établira que ce qu'un signe dénote est bien cet élément particulier qui provoque, de manière fiable, l'emploi du signe dans les circonstances favorables où les facteurs déstabilisants ne sont pas en opération. Le " rouge » réfère ainsi à la propriété qui fait que les choses ont l'air rouge et qui provoque, de manière fiable, les individus à dire " rouge » là où les facteurs du type illustré sont absents, autrement dit, lorsque les circonstances sont favorables à la perception du rouge.

Le fait que nous soyons des créatures discursives, au sens envisagé ici, signifie-t-il que notre expérience rendra les questions morales saillantes et 
qu'il sera nécessaire d'introduire des termes moraux ? Je soutiendrai qu'en tant que créatures discursives, il nous faut faire l'expérience de certaines réponses comme étant exigées normativement, et exigées normativement d'une manière qui sera saillante de la même façon pour nous tous. Mais comme je l'expliquerai dans la prochaine section, je pense que ces expériences ne nous donnent pas de concepts moraux proprement dits.

\subsection{Le normatif devient saillant}

Nous avons vu que les croyances et les désirs sont liés à certaines contraintes ou normes, en ce sens que nous ne les attribuons qu'à un sujet dans la mesure où nous constatons - à tout le moins dans des circonstances favorables et à l'intérieur des limites de faisabilité - que les états rencontrent des spécifications variables, nommément, des conditions variables d'entrée, de sortie et de performance. Or, si les croyances et les désirs sont ainsi liés à des normes de rationalité inférentielle, les phrases par lesquelles l'un d'entre nous exprime volontairement comment les choses se présentent à la lumière de ses croyances et désirs devront être en contact, et plus profondément, avec les normes correspondantes.

Considérons quelques-unes des conditions de rationalité inférentielle, qu'elles soient conclusives ou non conclusives, auxquelles des sujets intentionnels devront généralement se conformer, aussi aveuglément que ce soit. Le fait que la perception offre des raisons à l'appui que p exige, rationnellement, qu'un sujet intentionnel en forme la croyance correspondante et qu'il n'agisse pas sur la base du désir qui lui correspond : nul besoin d'agir à partir du désir que $\mathrm{p}$ si nous savons déjà que $\mathrm{p}$ est le cas. Le fait que le sujet croie que $\mathrm{p}$ et que si $\mathrm{p}$ alors q exige, rationnellement, qu'il croie que q. Le fait que le sujet désire que $\mathrm{p}$ et croie que par l'action $\mathrm{X}$ il peut faire en sorte que $\mathrm{p}$, exige qu'il fasse $\mathrm{X}$. Et ainsi de suite.

Or, si les croyances et les désirs des sujets intentionnels doivent satisfaire à certaines normes inférentielles, il en sera également ainsi des phrases par lesquelles des sujets discursifs tels que nous expriment ces croyances et désirs ; sinon, les phrases utilisées n'équivaudront pas à une expression sérieuse et sincère. Autant la croyance que $\mathrm{p}$ doit-elle être sensible aux raisons à l'effet que $\mathrm{p}$ ou non- $\mathrm{p}$, par exemple, autant l'usage assertorique de la phrase « $p$ » devra également être sensible à de telles raisons. Et autant le désir que $\mathrm{p}$ doit-il être sensible aux choses que l'agent croit et à celles vers lesquelles il est enclin, autant l'expression sincère et sérieuse du désir que $\mathrm{p}-$ disons, " la possibilité de $\mathrm{p}$ est attractive » — devra-t-elle être sensible à ces questions, questions qui, selon toute vraisemblance, sont susceptibles d'être exprimées par l'usage des phrases.

Nous pouvons concevoir que les croyances et désirs évoluent sur une base mécanique involontaire afin de s'ajuster aux exigences requises de la rationalité. Ils manifesteront ainsi une sensibilité aux exigences des normes 
inférentielles, mais sans que cette sensibilité ne soit contrôlée par des croyances au sujet de la nature de ces exigences. Par contre, nous ne saurions envisager que notre performance au moyen des signes volontaires fasse preuve d'une sensibilité tout aussi aveugle à l'égard des normes. Tandis que les croyances et désirs peuvent, chez des créatures intentionnelles, se matérialiser et subir une mutation d'une manière qui reste sensible à la norme mais qui n'est pas contrôlée par elle, rien de ce genre ne s'applique aux représentations linguistiques.

La raison en est que, à la base, le déploiement de ces phrases devra refléter la signification de nos croyances : ceci nous ramène, par conséquent, à cette hypothèse non acquittée selon laquelle nous avons accès au concept de signification. Et ces croyances, justement, représentent ni plus ni moins que des croyances au sujet des circonstances dans lesquelles nous devons ou non employer les signes. Après tout, connaître la signification en tant que telle d'un signe équivaut précisément à être capable de dire, avec une exactitude plus ou moins grande, quand son utilisation est juste ou non. Mais si nos phrases se déploient sous le contrôle de la signification de telles croyances, c'est donc dire que notre usage des représentations est contrôlé par les normes pertinentes, et non simplement sensible à ces normes ; il est guidé par nos croyances au sujet de ce que les normes exigent.

Considérons quelques-unes des normes inférentielles précédemment mentionnées, en laissant de côté les complications reliées au fait qu'on puisse les mettre en échec. Selon la première de ces normes, le fait que la perception offre des raisons à l'effet que p exige rationnellement qu'un sujet intentionnel forme la croyance correspondante. Tandis que des sujets intentionnels se conformeront de manière aveugle à cette condition en formant une croyance, des sujets discursifs souscriront plutôt à la représentation volontaire correspondante, qui est contrôlée par une croyance à l'effet que la perception les justifie de dire que $p$. D'après la seconde, le fait qu'un sujet intentionnel croie que $\mathrm{p}$ et que si p alors q exige, rationnellement, que celui-ci croie que q. Alors que des sujets intentionnels se conformeront de manière aveugle à cette condition en formant une croyance, des sujets discursifs souscriront plutôt à la représentation volontaire correspondante, celle-ci étant contrôlée par une croyance à l'effet que s'ils considèrent tout à la fois que $\mathrm{p}$ et que si p alors $\mathrm{q}$, ils sont justifiés de dire que q. De même, suivant une troisième de ces normes, le fait que le sujet désire que $\mathrm{p}$ et croie qu'en faisant $\mathrm{X}$, il peut faire en sorte que $\mathrm{p}$ exige, rationnellement, qu'il fasse $\mathrm{X}$. Tandis que des sujets intentionnels se conformeront de manière aveugle à cette condition lors du choix de l'action, chez des sujets discursifs, ce choix sera contrôlé par une croyance relative à l'aspect attractif de la possibilité de $\mathrm{p}$ et c'est cette connexion, entre faire $\mathrm{X}$ et la possibilité de $\mathrm{p}$, qui justifie que l'action $\mathrm{X}$ soit la chose à faire.

Lorsque nous utilisons, dans la pratique, ses signes volontaires ou une expression, nous devons, par conséquent, prendre conscience de certaines conditions normatives, c'est-à-dire des exigences de la rationalité inférentielle au sujet de notre performance. Autant nous sommes capables de pren- 
dre note de ce que les normes inférentielles exigent, autant il nous nous faudra, bien sûr, parvenir à réguler nos réponses comme les normes le requièrent. Autrement dit, nous devrons parvenir à réguler nos énoncés adéquatement, de même bien entendu que les croyances, désirs et actions qui leur sont associés. Sinon, nous ne pourrons pratiquer le discours avec succès ; il ne servirait à rien que quelqu'un nous parle. Nous devons être capables de penser que ceci ou cela est ce que nous devons dire et ce que nous devons croire, désirer ou faire. De plus, il nous faut rester en accord avec de telles pensées normatives, disant ce que nous pensons devoir dire et croyant, désirant et faisant ce que nous pensons devoir croire, désirer et faire. En bref, nous devons être capables de ratiociner ou de raisonner. En tous les cas, nous devons y parvenir sous des circonstances favorables et dans le cadre des contraintes propres à la faisabilité, peu importe comment on les identifie ${ }^{11}$.

Voici une dernière remarque pour préciser cette description. Être gouverné de cette manière par la raison reste compatible avec la possibilité que les signes que j'énonce soient déployés d'une manière relativement habituelle et non contrôlée. Il suffit que, malgré qu'une longue habitude ou une inertie règle les choses que je dis pour exprimer mes croyances ou désirs ou bien les choses que je fais sur la base de ces représentations, je puisse quand même réviser et amender, de manière intentionnelle, ce que je dis ou fais, dans le cas où une quelconque irrationalité inférentielle deviendrait apparente. Et l'on peut supposer que, dans les relations propres au discours, de telles irrationalités peuvent toujours devenir apparentes. En d'autres termes, la gouverne exercée par la raison peut être virtuelle plutôt qu'active. Il se peut bien que l'habitude explique plusieurs des choses que nous disons dans le discours, pourvu que la raison demeure en état de veille, prête à intervenir aussitôt qu'un défi est lancé à la rationalité inférentielle de ces énoncés ${ }^{12}$.

\section{Des sujets discursifs peuvent être dépourvus de concepts moraux}

Admettons que des sujets discursifs devront être conscients des exigences qu'imposent, sous différentes situations, les normes partagées de la rationalité inférentielle. Admettons que, dans cette mesure, ils devront forcément posséder des concepts normatifs. Ce qu'il nous faut maintenant remarquer, toutefois, c'est que le fait d'avoir accès de cette manière aux concepts normatifs n'implique pas que nous ayons un accès aux concepts moraux.

Les considérations normatives auxquelles des sujets discursifs doivent être sensibles et auxquelles ils doivent répondre sont des sortes de considé-

11. McGeer, V. et P. Pettit, "The Self-regulating Mind ", Language and Communication, 21, 2001.

12. Sur cette notion de contrôle virtuel, voir Pettit, P., " The Virtual Reality of Homo Economicus », Monist, 78, 1995, p. 308-329. 
rations qui expriment leurs croyances et désirs et qui soutiennent, inférentiellement, leurs croyances ou leurs désirs successifs de ceci ou cela, tout comme elles soutiennent leur façon d'agir selon ce que de tels croyances et désirs exigeraient. Des sujets discursifs pourraient, à partir du fait que $\mathrm{p}$ et que si $\mathrm{p}$ alors $\mathrm{q}$, déduire rationnellement que $\mathrm{q}$, et venir ainsi à croire que $\mathrm{q}$, s'ils ne le croyaient pas déjà. Partant du fait qu'en faisant X, ils peuvent produire que $\mathrm{p}$ et que la possibilité de $\mathrm{p}$ est elle-même attractive, ils pourraient en venir rationnellement à la conclusion que faire $\mathrm{X}$ est attractif, et en venir ainsi à désirer $\mathrm{X}$, s'ils ne le désiraient pas déjà. Ils pourraient déduire rationnellement, à partir du fait que $\mathrm{X}$, l'injonction d'accomplir $\mathrm{X}$ ou quelque chose de ce genre, et ainsi en arriver à accomplir X, et ainsi de suite. Les considérations impliquées serviront à fonder, de façon inférentielle, la formation des croyances et des désirs ultérieurs ainsi que l'accomplissement des actions.

Des sujets discursifs seront capables d'invoquer des normes communes de rationalité inférentielle afin de justifier de telles démarches aux yeux des autres, compte tenu des significations communes qu'ils attachent aux termes qu'ils utilisent. De telles démarches, peuvent bien entendu diverger de celles que les autres effectuent ou effectueraient à leur place. Mais s'il y a effectivement divergence, ce sera alors l'occasion de s'y arrêter et de rechercher une explication afin, idéalement, de résoudre le désaccord. On ne peut porter un regard insouciant sur les désaccords, comme s'ils étaient exempts d'erreur ${ }^{13}$. Ils doivent être considérés comme soulevant une question à propos de la rationalité inférentielle de l'une ou l'autre des parties impliquées.

Ce qu'il faut pourtant remarquer, à ce stade, c'est que tandis que les divergences en rapport aux croyances que forment les parties discursives envisagées ne peuvent être considérées comme exemptes d'erreur -du moins, pas si les croyances sont déterminées et non indexicales - celles impliquant leurs désirs seront certainement susceptibles d'être traitées de la sorte. Les sujets discursifs desquels nous avons parlé diffèrent des systèmes croyance-désir intentionnels par leur seule capacité à exprimer leurs croyances et leurs désirs de manière volontaire. Leurs croyances ne peuvent rationnellement diverger, compte tenu qu'une croyance a pour fonction de représenter comment sont les choses ; il en va donc de même quant aux expressions de croyance. Or, les désirs peuvent rationnellement diverger, compte tenu que le désir a une fonction directive plutôt que représentationnelle; ainsi en va-t-il également, par conséquent, des expressions d'un désir. Les sujets seront pressés de rechercher des raisons de s'accrocher aux croyances sur lesquelles ils sont en désaccord avec les autres, mais ils ne feront l'objet d'aucune pression visant à leur faire trouver des raisons de s'accrocher à leurs désirs particuliers divergeants. Ils n'ont peut être ni le besoin ni même l'habileté à faire quoi que ce soit qui puisse fournir des raisons de s'accrocher à leurs désirs particuliers.

13. L'idée d'un désaccord exempt d'erreur est tirée d'une notion que j'emprunte à Price, H., Facts and the Function of Truth, Oxford, Blackwell, 1988. 
La divergence qui est possible dans le domaine du désir, compte tenu de ce que suggère jusqu'ici notre analyse, peut être à ce point radicale que nous ne puissions considérer le désir que les mots et l'action d'autrui manifestent comme étant un désir intelligible auquel adhérer. Non seulement se peut-il que nous ne ressentions pas nous-mêmes le désir en question. Il se pourrait qu'il nous soit même impossible d'envisager comment nous pourrions être mus par ce désir si nous étions dans la position de l'agent. Le désir pourrait être aussi bête que l'inclination primaire et inintelligible que l'on retrouve dans l'exemple bien connu d'Elizabeth Anscombe, celle de posséder une soucoupe de boue ${ }^{14}$.

Il en découle que, tout en parvenant à exprimer leurs désirs de manière à offrir un fondement inférentiel à la formation de désirs subséquents et à l'accomplissement de certaines actions, des créatures discursives pourraient ne pas se montrer en mesure de résoudre les différences qui se présentent entre eux au niveau de leurs désirs primaires. Cette capacité à offrir des raisons qui soutiennent ces désirs pourraient leur faire défaut. Certes, ils peuvent essayer de résoudre les dilemmes, tant pratiques que théoriques, par une voie discursive, comme nous l'avions mentionné plus haut. Mais ils ne résoudront des dilemmes d'ordre collectif que s'il se trouve qu'ils ont des désirs qui convergent, et ils ne résoudront un dilemme d'ordre individuel que dans la seule mesure où ils tiennent les désirs de l'individu en question comme un donné non discutable. Les considérations qui expriment leurs désirs pourraient, d'une manière distinctive, servir de fondement aux actions, mais elles ne seront pas elles-mêmes supportées par des raisons; il ne pourra jamais être question de justifier les désirs exprimés en invoquant quelque chose qui fait de ces désirs ceux qu'un agent doit avoir.

Je résumerai ce résultat en indiquant que les créatures discursives envisagées jusqu'ici disposeront de concepts normatifs inférentiels mais qu'elles n'auront pas accès aux concepts moraux proprement dits. Une considération morale ou éthique est censée être à même d'offrir une raison inférentielle, conclusive ou non conclusive, à la formation d'un certain désir et à l'accomplissement de l'action correspondante : ceci, de façon à ce que la considération selon laquelle une chose est attractive - le type même de considération exprimant un désir - puisse offrir une raison inférentielle de la réaliser. Mais elle est aussi supposée être un type de considération telle que la divergence entre certaines parties à l'effet qu'elle soit ou non approuvée - une divergence portant, autrement dit, sur son statut de croyance - n'est pas une affaire de peu d'importance ; elle n'est pas nécessairement exempte d'erreur. Elle est censée être non seulement une considération fondant les actions, mais une considération elle-même fondée par des raisons.

14. Anscombe, G. E. M., Intention, Oxford, Blackwell, 1957 (trad. en partie dans Raisons pratiques : Les formes de l'action, Pharo, P. et L. Quéré, dir., Paris, EHESS, 1990, p. 257-266). 
Les considérations morales ne satisferont à cette condition double, c'est-à-dire tant celle d'être fondée par des raisons que celle de servir de fondement aux actions, que pour autant que l'une des deux choses suivantes est assumée en commun chez qui les invoque. Soit elles sont des considérations neutres par rapport à l'agent que chacun a quelque raison d'approuver; soit elles sont des considérations relatives à l'agent, mais que quiconque, se trouvant dans la position de cet agent, aurait quelque raison d'approuver. Du côté des considérations neutres par rapport à l'agent, on pourrait retrouver des exemples tels que "c'est la bonne chose à faire ", "c'est la seule réponse juste ", " c'est pour le bien général "; du côté des considérations relatives à l'agent, ces exemples seraient plutôt du type " cela me procurera du plaisir ", " cela aidera mes enfants» ou « ce sera pour le bien de mes amis".

Il n'est pas spécialement litigieux d'avancer que la divergence quant aux considérations morales n'est pas traitée comme exempte d'erreur ou affaire de peu d'importance chez ceux qui les invoquent. Le rôle joué par les considérations morales, celui de soutenir l'évaluation de ce que font les individus, ainsi que leur rôle connexe, à savoir permettre aux individus de justifier les uns avec les autres leurs désirs et actions, requièrent que la divergence soit vue comme suggérant l'erreur. Pour admettre ce point, nous n'avons pas à penser que chaque divergence morale se prête elle-même à une résolution mutuellement satisfaisante ; comme le soutiennent d'ordinaire les non-cognitivistes, il se peut qu'il y ait des valeurs fondamentales à propos desquelles les individus ne s'entendront pas et cela, d'une manière qui soit exempte d'erreur. La seule chose dont il nous faut convenir est que les considérations que les individus invoquent dans un registre moral invitent généralement à la discussion et offrent généralement une possibilité de réaliser un accord exempt de coercition et de collusion ; elles autorisent, généralement, une résolution discursive.

Par conséquent, nous aurons à chercher plus loin si nous voulons expliquer comment ce qui est moralement normatif, et non ce qui n'est qu'inférentiellement normatif, devient saillant pour nous. C'est ce à quoi je m'appliquerai dans les deux sections suivantes. Mais avant de passer à cette discussion, il importe de mentionner que les résultats de cette section-ci ne nous laissent pas dans un si grand embarras qu'on pourrait le croire. Bien que l'analyse ne puisse expliquer comment les termes ou les concepts moraux apparaissent, nous sommes arrivés beaucoup plus loin que le point auquel la discussion sur l'intentionnalité nous avait laissés.

J'ai soutenu plus tôt que des sujets simplement intentionnels n'auraient nullement la conscience en tant que telle de leurs perceptions et inclinations. Percevoir les choses sous un certain angle serait croire qu'elles sont ainsi ; être enclin à faire que les choses soient d'une certaine manière ne serait ni plus ni moins que de faire en sorte qu'elles soient ainsi, c'est-à-dire être disposé sans hésitation - le désir étant non conditionnel — à les faire ainsi. Ceci étant, il apparaissait que rien n'interpellerait de telles créatures à une prise de cons- 
cience de la perception comme offrant une raison à l'appui d'une croyance, ou bien de l'inclination comme offrant une raison à l'appui d'un désir (non conditionnel); c'est avec une force invisible, inéluctable, que la perception et l'inclination opéreraient dans la détermination de leurs croyances et désirs.

Ce qu'il faut remarquer à propos de la conception discursive des êtres humains, par opposition à celle simplement intentionnelle, c'est qu'elle n'est ni compatible avec le fait que la perception conserve un rôle silencieux, irrésistible dans la détermination des réactions individuelles, ni avec le fait que l'inclination se maintienne dans une position similaire. Elle exige que les participants puissent prendre conscience de leurs inclinations en tant que telles, et de celles des autres individus autant que des leurs.

Supposons que les individus utilisent des signes qui expriment comment le monde se présente à la lumière de leurs perceptions et disent, à partir de cette perception, que les choses sont comme ceci ou comme cela. Il apparaît alors inévitable que, tôt ou tard, certains ne s'entendront pas avec eux, considérant que ce sont d'autres aspects qui importent perceptuellement. Et cela veut dire que les individus ne pourront manquer de prendre conscience que leurs perceptions ne sont que des soi-disant représentations de la manière dont sont les choses, plutôt que de simplement parcourir leurs perceptions - sans être conscients qu'il s'agit de représentations - afin de prendre note que les choses sont de telle ou telle façon. Faire sens du désaccord avec autrui au moyen des hypothèses régulatives du discours obligera les individus à reconnaître leurs perceptions - et celles des autres - pour ce qu'elles sont : des représentations quant à la manière dont sont les choses, mais qui peuvent bien être loin de la vérité. Ceci ne pourra offrir une perception de la perception - aucune faculté extra-expérimentale ne se mettra en place - mais ceci les forcera à remarquer et à conceptualiser la perception qui était toujours déjà là. La vitre s'embuera, pour ainsi dire, cessant d'être un médium passé inaperçu, purement diaphane.

Il en sera d'ailleurs de l'inclination comme il en est de la perception. Selon l'analyse qui vient d'être présentée, le discours, au sein duquel les individus expriment leurs perceptions, leur fera comprendre que la manière dont se présentent les choses, dans le monde environnant, ne constitue pas un donné immédiat. De même, ce discours, au sein duquel les individus expriment leurs inclinations en discutant de ce qui est attractif ou non, leur fera comprendre que la manière dont le monde les incline à agir ne constitue pas, non plus, un donné incontesté ; ce n'est là que le produit d'une inclination, et c'est cela qu'ils devront être à même de constater. Auparavant, ils auraient parcouru leurs inclinations au sujet de la manière dont le monde se présente comme champ d'action, inconscients de ce qu'elles constituent en tant que telles. Or, ils seront maintenant sensibilisés au fait que le monde se présente comme un type de champ différent à des participants différents au discours. Pour rendre compte de cela, il leur sera nécessaire de reconnaître que la manière dont le monde incite l'un ou l'autre d'entre eux à l'action est fonc- 
tion des inclinations que chacun met au monde, et non le reflet de ce qui est disponible en commun pour tous.

Comme nous l'avions soutenu précédemment, aucune pression ne s'exerce sur les individus afin d'éviter une divergence d'avis au niveau des désirs non conditionnels, bien qu'une pression s'exercera afin d'éviter cette divergence à propos des croyances ultimes. Tout ce qu'il nous faut supposer, à cette étape de notre analyse, c'est que les individus peuvent généralement s'accommoder de la compétition et du conflit propres au domaine du désir ; ils ne pourront résoudre les dilemnes d'ordre pratique que dans la mesure où ils sont conduits par des questions de croyances sur lesquelles ils s'entendent et que les inclinations existantes convergent vers un désir commun. Le simple fait d'utiliser des signes pour exprimer comment le monde se présente à la lumière des inclinations devra suffire, à lui seul, à rendre visible l'inclination. C'est cela qui leur révèlera que le monde n'est pas un environnement générant l'action, avec lequel ils seraient en contact immédiat, pas plus qu'il n'est un environnement générant la croyance, avec lequel ils seraient en contact immédiat. Ils seront ainsi forcés de se considérer eux-mêmes et les autres non seulement comme des centres d'une représentation individuellement variable, mais aussi comme des centres d'une inclination individuellement variable.

Cette observation établit un élément négatif dans la généalogie des concepts moraux. Elle montre que les créatures discursives, à la différence des créatures purement intentionnelles, ne seront pas esclaves d'inclinations non détectées, ce qui exclurait l'apparition et l'à-propos des considérations morales. Mais il nous faut maintenant, à partir de cet argument négatif, tenter d'expliquer comment, et plus positivement, des créatures discursives en viennent à se montrer sensibles à des considérations morales, elles-mêmes susceptibles de réguler l'inclination et le désir. Je soutiens qu'il existe deux formes d'expériences qui se révéleront utiles aux créatures discursives à cet égard.

\section{Le privilège accordé au discours donnera lieu à certains concepts moraux}

Comme nous l'avons soutenu, dans la mesure où les individus entrent dans une situation de discours les uns avec les autres, ils devront prendre conscience de leurs propres inclinations en tant que telles et des inclinations rivales d'autrui. Mais comment des individus peuvent-ils en venir à accéder à des considérations qui guident l'action et qui ont la capacité de diriger l'inclination d'une manière distinctement morale ou éthique ? Quelles sont, selon le compte rendu discursif esquissé, les expériences à leur disposition qui sont susceptibles de donner lieu aux termes et concepts pourvus de cette double capacité spécifique au domaine moral, celle de servir de fondement aux actions et celle d'être fondé par des raisons ? Dans cette section, je décris une façon plus ou moins évidente par laquelle certaines expériences peuvent jouer ce rôle. Dans la section subséquente, je me dirige vers une autre voie possible. 
Selon la description présentée dans la section trois, les individus seront inévitablement engagés tantôt ici, tantôt là, dans un discours les uns avec les autres. Malgré que de nombreuses conversations serviront des objectifs autres, c'est-à-dire non discursifs, nous supposons néanmoins que la conversation est souvent vouée à la résolution de questions discursives, à savoir comment un certain dilemme pratique ou un certain dilemme théorique doit être résolu. Certaines de ces questions peuvent concerner ce que l'une ou l'autre des parties à la conversation doit penser ou faire; d'autres, ce que le groupe en tant que totalité - qu'il s'agisse d'un groupe de deux, trois ou plusieurs personnes - doit penser ou faire collectivement. Lorsque des individus prennent part à une telle conversation, ils se consultent et raisonnent les uns avec les autres sur la manière dont un dilemme pratique ou un dilemme théorique doit être résolu.

Un tel discours renferme les normes rationnelles inférentielles reconnues, lesquelles indiquent, eu égard à la manière dont les choses sont présentées sur le plan perceptuel, le moment où il apparaît approprié de souscrire à une considération donnée, ou de souscrire à deux ou plusieurs considérations en même temps, ou de passer de l'une ou des considérations approuvées vers l'approbation d'une considération supplémentaire, ou, enfin, de passer de l'approbation de certaines considérations à l'exécution de telle action ou de telle autre. Nous ne pourrions considérer les autres comme partenaires discursifs s'ils n'étaient d'ordinaire capables de reconnaître et de répondre à ce qu'exigent de telles normes. Devaient-ils ainsi échouer à internaliser les normes discursives inférentielles, nous ferions alors tout aussi bien de parler au chien ou au mur ${ }^{15}$.

Mais si une conversation discursive de ce type doit être gouvernée par les normes rationnelles inférentielles du discours, il s'ensuit que ceux qui y prennent part devront aussi se conformer aux normes interactives du discours. Si vous et moi raisonnons ensemble à propos de quelque chose, alors la conversation discursive dans laquelle je suis engagé exige, de par sa nature même, que les ouvertures que je fais soient toutes compatibles avec notre intention commune, celle d'être gouvernés par des normes rationnelles inférentielles selon la manière précédemment envisagée. Par conséquent, si j'avance une considération que je sais être fausse, mon comportement n'est pas en accord avec cette intention. Si j'exprime, de façon inauthentique, une intention quant à ce que je ferai dans l'avenir, suggérant par ce biais une résolution discursive d'un dilemme pratique, mon comportement n'est pas en accord avec cette intention. Si j'adresse une menace coercitive à l'effet que, tant que les autres ne s'entendront pas avec moi à propos de quelque chose, je pourrai les malmener, mon comportement n'est pas en accord avec cette intention, et ainsi de suite. Raisonner ensemble est un exercice qui, de par sa nature même, est incompatible avec l'adoption, chez les parties engagées, de

15. Pettit, P. et M. Smith, "Freedom in Belief and Desire », p. 429-449. 
tels modes de conduite. Si nous laissons l'une des parties se comporter ainsi, ce qui en sortira ne pourra jamais être décrit comme l'exemple d'un raisonnement en commun à propos d'une question discursive.

Les individus ne devront pas seulement se conformer aux normes interactives de la conversation discursive, c'est-à-dire aux normes dérivant des normes, beaucoup plus fondamentales, de type inférentiel. Les individus qui prennent part à la conversation discursive devront aussi être conscients de ce qu'exigent ces normes interactives, tout au moins au cas par cas, et ils devront faire la preuve qu'ils sont en mesure de répondre à ces conditions. La capacité à prendre part à une telle conversation est une habileté que les individus apprennent, non quelque chose qui survient comme un automatisme ou un réflexe. Au cours de l'apprentissage de cette habileté, il est en outre essentiel qu'ils soient à même de reconnaître et de rejeter les démarches qui sont de l'ordre de la tromperie, de la manipulation et de la coercition, lesquelles sont incompatibles avec l'exercice du raisonnement avec autrui, visant à résoudre une question théorique ou pratique. Tromper, manipuler ou contraindre les autres, chacun reste, bien sûr, libre de le faire ou non. Mais tous devront être à même de constater que les options de la tromperie, de la manipulation ou de la coercition sont incompatibles avec le maintien d'une posture discursive face à autrui. Et bien sûr, ils devront être à même de se conformer à cette perception, en se montrant capables de traiter autrui comme un semblable qui raisonne.

Mais si les individus doivent être capables de reconnaître certains types d'ouverture comme incompatibles avec la posture discursive - la posture de la conversation mutuelle, comme Stephen Darwell l'appelle ${ }^{16}$ — c'est donc qu'ils doivent être en position d'introduire des termes ayant une force éthique afin de décrire les options en question. Acceptons ici que les termes introduits soient représentés par la lettre « $\mathrm{O}$ » (qui signifie ouvert aux objections). Dans la mesure où le discours et la conversation sont des pratiques admises par les individus, la considération selon laquelle une option est $\mathrm{O}$ aura le profil d'une considération morale, à savoir, d'une considération qui peut servir de fondement aux actions et être fondée sur des raisons.

La considération selon laquelle une option est $\mathrm{O}$ ne servira de fondement aux actions que pour autant qu'elle exprime un engagement volontaire et non conditionnel à la pratique conversationnelle ou discursive, laquelle, en premier lieu, rend disponible le concept de type O. Penser - alors que l'on se trouve soi-même dans une situation de discours - qu'une certaine sorte d'action est $\mathrm{O}$, ce sera prendre note d'une raison, conclusive ou non conclusive, pour se soustraire à une telle ouverture. Ce sera en quelque sorte penser, alors que l'on soutient un certain désir ou inclination, que ce qui est incompatible avec la satisfaction de ce désir est non attractif.

16. Darwall, S., Reciprocal Recognition: The Second-Person Standpoint in Moral Thought and Theory, Ann Arbor, Dept. of Philosophy, University of Michigan, 1999. 
Mais la considération selon laquelle une option est $\mathrm{O}$ sera aussi susceptible d'être fondée par des raisons, à la différence, par exemple, de cette considération suivant laquelle ce qui est incompatible avec la possession d'une soucoupe de boue est non attractif. C'est qu'il n'y a rien qu'une personne puisse faire en vue de soutenir le désir primaire pour une soucoupe de boue. Rien à propos de ce désir, c'est du moins ce que nous avons supposé, n'est susceptible d'en faire quelque chose qu'il serait bon, dans l'une ou l'autre des acceptions de ce terme - c'est-à-dire bon pour le sens commun — de vouloir endosser. C'est pourtant de façon tout à fait différente que les choses se présentent lors d'un engagement discursif qui présuppose que les considérations du type $\mathrm{O}$ soient traitées comme guidant l'action. Un tel engagement ne peut d'ailleurs manquer de se recommander généralement aux individus, compte tenu de leur nature discursive. Toute personne sera ainsi en position de justifier sa considération que le fait qu'une option est $\mathrm{O}$ est une raison pour se soustraire à l'action, par le fait - peu importe la façon dont on le formule - que c'est justement un traitement de ce type qui est exigé afin de maintenir la position discursive et conversationnelle.

Les prédicats du type $\mathrm{O}$ apparaîtront comme tributaires du discours ou de la conversation de l'une ou l'autre de deux manières possibles, selon ce qu'illustre l'observation suivante. Quelqu'un qui considère qu'une option est O peut d'abord vouloir dire, dans une perspective neutre, que l'option est incompatible avec la conversation ; elle serait exclue par la pratique discursive de la conversation mutuelle. Ou encore, occupant la perspective de la conversation mutuelle - et considérant, par ce moyen que la pertinence de l'option est sans rapport avec la question présente - cette personne peut vouloir dire qu'elle est exclue, point final : elle serait tout simplement exclue, quoique peut-être seulement de manière non conclusive plutôt que conclusive. La première interprétation donnerait un caractère relationnel aux termes de type $\mathrm{O}$; la seconde, un caractère perspectiviste ou situationnel.

Je suppose que c'est de manière situationnelle que les termes dits $\mathrm{O}$ seront tributaires de la conversation. Cette interprétation est plus économique que celle dite relationnelle, puisqu'elle n'exige pas que les individus disposent d'un concept explicite du discours et de la conversation, mais seulement qu'ils soient engagés d'une manière volontaire et non conditionnelle dans cette pratique. En raison du fait qu'ils occupent une position discursive, les individus considéreront certains types d'ouvertures $\mathrm{O}$, mais sans peut-être remarquer que c'est précisément en vertu de leur rapport au discours que ces ouvertures se présentent comme O. Cette position discursive jettera une ombre, pour ainsi dire, sur les types d'action pertinents, les rendant ainsi disponibles, d'une manière saillante, à une conceptualisation en tant que $\mathrm{O}$, et cela, sans que les individus aient nécessairement pris conscience du rôle joué par la position discursive au cours de ce processus.

Considérer que les termes dits $\mathrm{O}$ sont de caractère situationnel plutôt que relationnel présente, à côté de son souci d'économie, un autre avantage. 
C'est que, sous cette interprétation, on peut rendre compte du fait que les actions accomplies à l'extérieur du contexte de la conversation discursive soient considérées comme $\mathrm{O}$. Autrement dit, cette interprétation contribue à élargir le domaine au sein duquel ces termes peuvent être appliqués de manière non tendancieuse. Reprenant une position discursive, je peux penser que quelque chose que je ferais à une certaine personne serait $\mathrm{O}$, et cela même si je n'ai aucun intérêt à converser avec cette personne affectée ; en pensant ainsi, je privilégierai la position discursive que j'occupe présentement, utilisant celle-ci comme ligne de fond pour évaluer le comportement que je conçois. Et tout comme je peux penser ce genre de choses à propos de l'action que je pourrais moi-même accomplir à l'extérieur du contexte de la conversation, autant puis-je en penser des actions que d'autres accomplissent présentement à l'extérieur d'un tel contexte. Dire de certaines actions qu'elles sont interdites par la conversation discursive inviterait la réponse suivante : «Et alors? Ces personnes ne pratiquent pas présentement la conversation ». Dire, à propos de telles actions, qu'elles sont interdites, point final, n'autorisera pas une réponse aussi facile, même si la remarque devait être adressée à partir d'une position privilégiant le discours et la conservation.

Les éléments nécessaires à une généalogie des concepts moraux sont maintenant en place. Le discours implique quelque chose qui est, espéronsnous, incontestable d'un point de vue naturaliste, à savoir, l'échange de signes communs en vue d'exprimer volontairement des états intentionnels. À partir de leur position discursive, les participants devront considérer l'utilisation de ces signes comme sujette à des normes rationnelles inférentielles, comme nous l'avons soutenu dans la dernière section. En outre, et tel que nous l'avons soutenu dans cette section-ci, ils devront considérer les activités impliquant l'échange discursif des signes - activités pouvant être ou non en accord avec la pratique du discours et de la conversation - comme sujettes aux normes interactives et aux considérations morales correspondantes.

À mes yeux, l'analyse livrée peut se révéler convaincante pour quiconque accepte la conception discursive esquissée dans la seconde section. Et quand bien même elle se révélerait erronée sous certains rapports, elle pourrait encore servir à rendre intelligible la conceptualisation éthique. Que les individus puissent se sensibiliser aux considérations morales de la manière ici décrite établit, à tout le moins, qu'il n'y a rien de mystérieux en soi, même selon des présupposés largement naturalistes, dans le fait d'avoir accès aux termes et concepts moraux. Même en admettant que l'explication soit de caractère tout à fait hypothétique, elle servirait encore à rendre intelligible l'apparition des termes et concepts éthiques ${ }^{17}$. Donald Davidson affirmait rendre compte de l'intelligibilité de notre compétence sémantique à l'égard d'un nombre indéfini de phrases en montrant qu'elle aurait pu surgir — bien

17. Elle serait entièrement hypothétique, selon l'analyse présentée dans Brandom, R., Making it Explicit, Cambridge (Mass.), Harvard University Press, 1994. 
qu'en fait ce ne soit certainement pas le cas - de l'apprentissage, pour la langue considérée, d'une théorie tarskienne de la vérité ${ }^{18}$. Nous pourrions affirmer que l'analyse tout juste présentée, quand bien même elle ne serait pas fidèle à l'évolution réelle des concepts moraux, peut rendre intelligible, d'une manière semblable, la conceptualisation morale.

Pour éviter de clore cette discussion relative aux concepts tributaires de la conversation sur une note faible, j'aimerais souligner combien la conversation discursive caractérise et met en valeur la vie humaine et jusqu'à quel point il est naturel qu'elle puisse offrir une entrée à la discussion et à la pensée éthiques. Il est possible pour une personne, au cours d'un échange discursif, d'exercer une influence substantielle auprès des autres, en se montrant mieux informée, plus perspicace, plus persuasive ou que sais-je encore. Mais l'influence qui s'exerce de cette manière est tout à fait différente d'une influence trompeuse, manipulatrice ou coercitive, ou encore de toutes ces sortes d'influence que l'on retrouve dans le monde sensible. Elle est d'un genre tel qu'elle doit être bien accueillie par les personnes influencées, dans la mesure où elles sont sincèrement intéressées par la question discursive qui est sous examen. En outre, elle est un genre d'influence qui, en aucune façon, ne porte atteinte à la capacité d'une personne à être tenue responsable de son agir ; elle ne mine ni ne diminue son autonomie en tant qu'agent ${ }^{19}$. Si c'est là le lieu où les termes et concepts éthiques acquièrent, en premier lieu, une existence, il est aussi adéquat que tout autre lieu que nous pourrions jamais concevoir.

\section{L'exercice discursif du sentiment donnera lieu à d'autres concepts moraux}

Si l'on se rapporte à l'analyse qui vient d'être présentée, les individus considèrent la pratique du discours comme donnée et, à partir de la perspective privilégiée ainsi offerte, ils distinguent les activités dissonantes d'une conversation discursive de celles qui lui sont consonantes, en les identifiant comme O, c'est-à-dire comme ouvertes aux objections. En privilégiant le discours, c'est donc à la position discursive qu'ils laissent le soin de départager les activités qui sont $\mathrm{O}$ de celles qui ne le sont pas.

Mais pouvons-nous envisager comment des individus pourraient développer des termes et concepts éthiques, mis à part les concepts tributaires de la conversation que ce privilège accordé au discours explique ? Une autre source, propre à la conceptualisation éthique, peut-elle être identifiée à partir de l'analyse précédemment rapportée ? Nous avons déjà mentionné dans les discussions précédentes qu'une personne peut considérer le caractère attractif

18. Davidson, D., Inquiries into Truth \& Interpretation, Oxford, Oxford University Press, 1984 (Enquêtes sur la vérité et l'interprétation, trad. P. Engel, Nîmes, Éd. Jacqueline Chambon, 1993). En français dans le texte.

19. Pettit, P., A Theory of Freedom : From Psychology to Politics, Cambridge, Polity, 2001. 
d'une option comme représentant une raison inférentielle de la choisir, sans que cette considération soit elle-même fondée de manière à assurer sa force éthique ; autrement dit, sans qu'il n'y ait aucune raison, accessible à l'agent, en vertu de laquelle il devrait l'approuver comme raison d'agir. La question est maintenant de savoir si les individus peuvent escompter développer des concepts au moyen desquels de telles considérations pourraient être investies d'un statut qui fonde les actions tout en étant aussi fondées par des raisons, selon le modèle des considérations tributaires de la conversation.

À mes yeux, nous pouvons escompter que les individus développent de tels concepts. Ils auront des raisons suffisantes de le faire, attendu qu'une fois entrés dans l'espace éthique - une fois venus à considérer la possibilité d'une régulation de l'inclination sur la base de considérations auxquelles ils souscrivent mutuellement - ils ne pourront manquer d'être sensibles à la possibilité de la coordination plus riche qu'offriraient des concepts éthiques supplémentaires. Et de même qu'il existe une raison qui incite les individus à essayer de développer des concepts éthiques supplémentaires, ils auront aussi une ressource à leur disposition qui les aidera à réaliser ce but.

Pour toute option qu'un agent pourrait considérer comme attractive, les individus seront en position de soutenir la raison fondant l'action en question - la présence de l'inclination — dans la mesure où ils convergent sur les critères indiquant quand il est approprié de former une inclination et d'agir sur cette base. Ceci laisse entendre, en outre, qu'ils seront en position de soutenir la raison fondant l'action, fournie par une inclination quelconque, en autant qu'ils convergent sur la propriété que l'inclination est généralement censée détecter. La possibilité de découvrir des critères, indiquant quand l'embarras ou la peur sont appropriées, par exemple, est précisément la possibilité de découvrir la propriété - celle d'être réellement embarrassant ou réellement effrayant, pourrions-nous dire — qu'elle est censée détecter.

Cette observation attire notre attention vers une procédure de recherche au moyen de laquelle les individus pourraient aspirer à discriminer les inclinations susceptibles de générer des considérations d'ordre éthique. Comme cadre de travail, les individus pourraient recourir à la supposition qu'un sentiment donné, disons l'embarras ou la peur, possède un statut particulier, celui de représenter quelque chose qui nous est disponible en commun à tous c'est-à-dire ce que serait, véritablement, l'embarrassant ou l'effrayant — afin de constater jusqu'où une telle supposition s'avère applicable. La supposition s'avérera applicable si les individus constatent d'ordinaire qu'il est possible qu'il y ait une convergence au niveau de leurs réactions et que, là où il y a divergence, celle-ci puisse être résolue ou expliquée d'une manière qui soit compatible avec le fait que le sentiment ait un statut représentationnel. La supposition se révélera applicable dans le cas de la peur, par exemple, dans la mesure où les individus tendent à qualifier les mêmes scénarios d'effrayants et qu'ils se trouvent capables, dans le cas d'une divergence, de s'entendre afin de déterminer laquelle de ces réponses contradictoires devrait être rejetée. 
La procédure de recherche envisagée peut être caractérisée, de manière plus précise, au moyen de termes abstraits. Supposons que les individus tendent à ressentir un sentiment $S$, en se conformant à un modèle relativement commun ; autrement dit, ils réagissent à des stimuli approximativement identiques sous des conditions approximativement identiques. Les modalités au moyen desquelles ils pourraient tester la capacité de $S$ à être fondée par des raisons et à jouer un rôle éthique pourraient être les suivantes. Supposons qu'ils introduisent un prédicat « $\mathrm{D}$ » (pour désirable) comme une expression de $S-$ celui-ci correspondant à l'effrayant dans l'expression de la peur -, et voyons s'il leur est possible de le soumettre, de manière effective, aux trois hypothèses régulatives qu'implique une pratique commune discursive. Laissons-les voir si le prédicat $\mathrm{D}$ peut se prêter à un exercice discursif, lequel lui permettrait de fonctionner sur un plan parallèle aux prédicats discursifs réguliers. Ce qui devra être testé, au cours de l'exercice, c'est la question de savoir si, en considérant $\mathrm{D}$ comme une expression du sentiment $\mathrm{S}$, ils peuvent encore réguler leur pratique avec succès au moyen de ces trois hypothèses qui stipulent, premièrement, qu'il y a une propriété, soit neutre par rapport à l'agent soit relative à l'agent, à savoir $\mathrm{D}$, laquelle est en attente d'être détectée au moyen de « $\mathrm{D}$ »; deuxièmement, que chacun a cette intention commune, en utilisant $\mathrm{D}$, de détecter la même propriété supposément saillante ; et troisièmement, que chacun est d'ordinaire compétent dans ses tentatives visant à détecter $\mathrm{D}$.

Réfléchissons à la manière dont le processus pourrait se dérouler. Il sera nécessaire que les individus utilisent « $\mathrm{D}$ » pour exprimer $\mathrm{S}$, et ceci avec l'intention d'en marquer une propriété, laquelle offre une raison d'attribuer $\mathrm{D}$ et de soutenir S. Pour autant qu'ils trouvent un moyen de converger dans l'usage des termes, tout se déroulera correctement. Et quand bien même il y aurait une divergence d'avis, tout se déroulera encore correctement s'ils peuvent maintenir les trois hypothèses régulatives. C'est ce qu'ils seront à même de faire, par la suite, dans la mesure où ils en viennent à s'entendre, au cas par cas, sur les types de facteurs qui déstabilisent la performance et qui donnent lieu au désaccord. C'est ce qu'ils seront à même de faire, en fait, tant qu'ils ont des moyens communs d'identifier certaines conditions - appelons-les « $\mathrm{C} »-$ exemptes de telles influences déstabilisantes, lesquelles se montrent ainsi susceptibles de détecter la présence de la propriété, c'est-àdire $\mathrm{D}$, et d'approuver le sentiment, c'est-à-dire $\mathrm{S}$.

Suivant cette description, la question de savoir si quelque chose est $\mathrm{D}$ ou non sera en réalité indépendante du fait que le sujet ressente $S$ ou non. C'est pourquoi cette description peut faire une place pour la réflexion, selon laquelle la considération formulée par " $\mathrm{D}$ ", et qui doit offrir à l'agent une raison inférentielle d'agir selon ce que provoque $S$, peut être digne ou non d'approbation. Dans la mesure où les limitations, obstacles, défaillances cognitives et autres phénomènes de ce genre sont possibles - dans la mesure où les conditions dites $\mathrm{C}$ peuvent bien ne pas être obtenues - il sera possible à une personne de ressentir $\mathrm{S}$ alors que ce sentiment est rationnellement exclu 
et de ne pas l'éprouver alors qu'il semble rationnellement justifié. Il sera donc possible à des individus de ressentir $S$ alors que l'objet de leur sentiment n'est pas $\mathrm{D}$ - comme ils pourraient être à même de le constater — et de ne pas ressentir $\mathrm{S}$ alors que l'objet de leur sentiment est $\mathrm{D}$ - comme ils pourraient encore être à même de le constater. Le lien entre le sentiment $\mathrm{S}$ et la qualité $\mathrm{D}$ ne sera pas d'un type tel qu'une chose possède la qualité $D$ si et seulement si elle produit $S$ chez la personne appropriée. Plutôt, une chose possédera la qualité $\mathrm{D}$ si et seulement si elle est à même de produire $\mathrm{S}$ chez une personne appropriée sous des conditions dites $\mathrm{C}$, c'est-à-dire dans des circonstances telles que la réaction de la personne n'est en aucune façon susceptible d'être déstabilisée.

On devrait noter que cette analyse est tout à fait compatible avec le fait que les individus considèrent la qualité $\mathrm{D}$ comme une caractéristique du monde en tant que tel ou de la manière dont il exerce un impact sur l'agent, sans qu'ils prennent note du lien qui la relie au sentiment. De même que les termes dits $\mathrm{O}$ peuvent être tributaires de la conversation sans être des termes relationnels qui expriment une relation incompatible avec la conversation, les termes dits $\mathrm{D}$ peuvent être tributaires des sentiments sans être des termes relationnels qui expriment la relation qui consiste à occasionner un sentiment. Comme dans le cas des concepts tributaires de la conversation, il est plus économique d'admettre que ces concepts tributaires du sentiment sont perspectivistes ou situationnels, puisque cela laisse entendre que le fait de saisir et le fait de conceptualiser les propriétés dites D n'exigera pas une saisie et une conceptualisation préalable des sentiments dits $S$ ou des conditions dites C. Ceux qui utilisent les termes D n'ont pas besoin de distinguer, en tant que classe générale, les conditions $\mathrm{C}$ ou bien d'identifier ou de nommer, dans leur for intérieur, la réaction $S$, ni encore d'être conscients du fait que ces choses qu'ils appellent « $\mathrm{D}$ » donnent lieu à $S$ sous les conditions $C^{20}$. La supposition selon laquelle les termes $\mathrm{D}$ sont situationnels a aussi pour effet d'élargir le domaine potentiel au sein duquel ces termes peuvent s'appliquer de manière non tendancieuse. Je peux penser que la chose que je ferais serait $\mathrm{D}$, même si, à ce moment, je ne devais pas ressentir ou approuver le sentiment $S$; et je peux penser qu'il en va également ainsi des autres qui agissent présentement de cette manière, et même de la part de ceux qui ne ressentent ni n'approuvent le sentiment.

Quels pourraient être des exemples de termes dits D ? Certains seront des termes qui répondent, de manière déterminante, à des sentiments reconnaissables, comme c'est le cas de "l'effrayant ", " l'embarrassant », « le palpitant ", "le dégoûtant ", "l'humiliant " et "l'inspirant ». D'autres termes seront similaires à ceux-ci, à l'exception près qu'ils marqueront, plus explicitement, le fait qu'il est possible de ressentir le sentiment sans que le prédicat s'applique tout comme il nous est possible, bien sûr, d'être effrayé, sans qu'il n'y ait rien

20. Pettit, P., «Realism and Response-Dependence », Mind, 100, 1991, p. 587-626;

" Terms, Things and Response-Dependence », European Review of Philosophy, 3, 1998, p. 55-66. 
de réellement effrayant - et vice-versa. C’est dans cette seconde catégorie que se retrouvent des termes tels que " admirable ", " honorable ", "désirable " ainsi que leurs opposés négatifs tels que " abominable ", " déshonorant ", " indésirable ». D'autres termes encore ne se concentreront ni sur le sentiment à l'origine d'une réaction ni sur le fait qu'il peut être soutenu ou non avec justesse, mais plutôt sur le type d'action, de personne ou d'état de choses qui suscite le sentiment positif ou négatif en question. C'est dans cette troisième catégorie que se retrouvent les termes plus substantiels au niveau descriptif, tels que " gentil » ou " cruel », « juste » ou « injuste », «courageux » ou " lâche ", " prudent » ou «imprudent », et ainsi de suite.

Remarquons que, parmi ces exemples de termes dits $\mathrm{D}$, nous devrions nous attendre à trouver des termes qui prennent en note des aspects de l'expérience et de l'interaction, lesquels sont tributaires de la pratique du discours elle-même et du développement préalable des termes dits O. Pour autant que ce qui présente un aspect $\mathrm{O}$ devient saillant à la lumière de la pratique discursive, et dans la mesure où cette dernière est plus ou moins stimulante, elle ne peut manquer d'impliquer nos sentiments; elle impliquera, plausiblement, les sentiments phénoménologiquement distinctifs que sont le ressentiment et l'indignation et sur lesquels Peter Strawson a attiré l'attention ${ }^{21}$. Et dans la mesure où ces ouvertures qui se présentent comme $\mathrm{O}$ - la tromperie, la manipulation, la coercition, etc. - héritent des caractéristiques de ce qui présente un aspect $\mathrm{O}$, elles seront à même de susciter des sentiments autonomes de désapprobation. Nous devons être prêts à admettre qu'il y aura des interactions inévitables de ce genre entre les concepts éthiques tributaires de la conversation et ceux qui sont tributaires du sentiment.

Nous avons vu pourquoi les termes et concepts qui se conforment au schéma $\mathrm{D}$ devraient posséder cette capacité double, laquelle est la marque distinctive du domaine éthique. Les considérations D étant l'expression de sentiments dont le composant attractif exprime une inclination offriront des raisons inférentielles, conclusives ou non conclusives, pour l'action. En outre, les considérations D seront fondées par des raisons, pour autant qu'il sera raisonnable de les approuver dans ces cas où elles ont vraiment cours. Alors que les considérations $\mathrm{O}$ seront probablement neutres par rapport à l'agent, présentant des raisons auxquelles nous avons tous une quelconque raison de souscrire, les considérations $\mathrm{D}$, elles, seront relatives à l'agent aussi souvent qu'elles sont neutres par rapport à l'agent. Ces dernières incluront tant des considérations qui ont trait aux enfants ou aux projets pour lesquels l'agent a un sentiment spécial — c'est-à-dire, un sentiment qui lui appartient en propre - que des considérations liées à des problématiques à propos desquelles nous pouvons escompter ressentir approximativement les mêmes sentiments.

21. Strawson, P., "Freedom and Resentment", dans Walston, G. dir., Free Will, Oxford, Oxford University Press, 1982 ( « Liberté et ressentiment «, dans Neuberg, M., dir., La responsabilité, Paris, PUF, 1997, p. 109-140). 
L'analyse des termes $\mathrm{O}$, livrée dans la dernière section, explique comment des créatures telles que nous, pour autant que nous pratiquions le discours et privilégions la position conversationnelle, reconnaissons certaines activités, sur le plan moral, comme étant ouvertes aux objections ou, évidemment, comme étant incontestables. L'analyse des termes $\mathrm{D}$ joue un rôle généalogique similaire. Elle explique comment des créatures discursives telles que nous, qui se sont déjà familiarisées avec les concepts éthiques, peuvent privilégier certains sentiments - ceux reconnus comme se prêtant à un exercice discursif - et ressentir les objets de ces sentiments comme désirables ou indésirables moralement. Sous l'analyse présentée, un X quelconque sera $\mathrm{D}$ si et seulement si $\mathrm{X}$ a une disposition à produire $\mathrm{S}$ dans des conditions $\mathrm{C}$. Ce que cette formule nous communique, c'est que les concepts du type $\mathrm{D}$ seront disponibles, sous un mode canonique ou non parasitaire, à ces seules créatures qui sont capables d'une réponse $\mathrm{S}$ et qui soumettent cette réponse à un exercice discursif, en ce sens où ce n'est que dans ces strictes conditions avérées $C$ qu'ils s'y tiendront.

C'est donc une conclusion identique qui doit s'appliquer aux résultats de la seconde généalogie : comme la première, elle peut éclairer la possibilité d'une conceptualisation éthique même si elle devait ne pas être exacte dans le détail. Il importe pourtant de mentionner qu'il existe une longue tradition en faveur de cette seconde généalogie et que, pour cette raison, elle peut être considérée comme ayant des prétentions particulièrement fortes. Cette généalogie se conforme à une narration du sentiment et à un apprentissage des sentiments, lesquels étaient d'acceptation commune au cours du siècle des Lumières et sont aujourd'hui mieux connus par les travaux de David Hume $^{22}$. En outre, elle s'accorde avec les travaux produits, dans le domaine méta-éthique, par ces auteurs contemporains qui restent fidèles, dans ses grandes lignes, au cadre conceptuel huméen ${ }^{23}$.

22. Hume, D., Of the Standard of Taste and Other Essays, John Lenz, dir., Indianapolis, Bobbs-Merrill, 1995 (Essais esthétiques, trad. J. Bouveresse, Paris, Vrin, 1971); A Treatise of Human Nature, Nidditch, P. H., dir., Oxford, Oxford University Press, 1978 (Traité sur la nature humaine, trad. A. Leroy, Paris, Aubier, 1983); An Inquiry Concerning the Principles of Morals, Schneewind, J. B., dir., Indianapolis, Hackett, 1993 (Enquête sur les principes de la morale, trad. A. Leroy, Paris, Aubier, 1947). Voir aussi, Sayre-McCord, G., " On Why Hume’s "General Point of View» Isn't Ideal - and Shouldn't Be », Social Philosophy and Policy, 11, 1993, p. 202-228 ; Darwall, S., The British Moralists and the Internal 'Ought', Cambridge, Cambridge University Press, 1995 ; et Schneewind, J.B., The Invention of Autonomy, Cambridge, Cambridge University Press, 1995. Parmi les auteurs qui insistent sur la possibilité d'une lecture cognitiviste de Hume, de telle sorte que son approche pourrait refléter celle adoptée ici, on retrouve Mackie, J. L., Hume's Moral Theory, London, Routledge, 1980 ; Sainsbury, R. M., «Projections and Relations », Monist, 81, 1988, p. 133-160 ; Railton, P., "Taste and Value », dans Crisp, R. et B. Hooker, dir., Well-being and Morality: Essays in Honour of James Griffin, Oxford, Oxford University Press, 2000.

23. Pour un développement de cette approche dans une perspective moderne, voir, par exemple, Smith, M., The Moral Problem, Oxford, Blackwell, 1994. Or, si l'approche de Smith accorde une grande importance à la notion du point de vue idéal, on pourrait toutefois soutenir que c'est plutôt sur la notion du point de vue général que repose la position défendue par Hume. 


\section{Conclusion}

Si l'épistémologie de l'éthique est appelée à expliquer comment les jugements éthiques peuvent être confirmés, elle a aussi pour tâche d'aborder cette autre problématique, c'est-à-dire chercher à rendre compte, à partir de l'expérience, de l'origine de la conceptualisation éthique. L'expérience des sujets intentionnels ne saurait donner lieu à une conceptualisation morale en tant que telle, mais on ne peut nier que c'est bien ce à quoi parviendra l'expérience des créatures discursives. Cette expérience occasionnera inévitablement l'apparition de certains concepts normatifs, puisque ceux-ci sont nécessaires au raisonnement. En outre, elle se révélera également à même de donner lieu à une véritable conceptualisation morale. Elle y parviendra, en premier lieu, en rendant saillantes les exigences présupposées par la pratique de la conversation discursive, et, en second lieu, en encourageant l'exercice discursif du sentiment.

Ces deux analyses d'ordre généalogique demeurent, l'une et l'autre, suffisamment convaincantes à mes yeux, bien que chacune demande encore à être développée plus en détail. Les distinguer est une chose importante, car les concepts tributaires de la conversation et les concepts tributaires du sentiment qu'elles contribuent respectivement à étayer demeurent de caractère tout à fait différent. Toute théorie morale compréhensive aura à reconnaître leur importance spécifique et à expliquer comment les différentes exigences en question doivent être réconciliées. Et dépendamment de l'ampleur avec laquelle on priorisera l'un ou l'autre type de concept, la théorie prendra tantôt une certaine forme, tantôt une autre.

Les concepts tributaires de la conversation demeurent, grosso modo, de caractère déontologique. La question à laquelle ils se rapportent a pour objet de déterminer si quelque chose constitue un manquement aux normes discursives - autrement dit, si une chose est deon ou en dette envers autrui - et elle ne s'applique qu'aux seules actions que les individus entreprennent dans leurs relations les uns avec les autres. Il est possible de traiter de telles considérations négatives, axées sur l'action, d'une manière conséquentialiste plutôt que non conséquentialiste : on pourrait penser, par exemple, que l'action juste exige de maximiser ce type de comportements qui, vis-à-vis des autres, n'est pas ouvert aux d'objections, même si elle n'exige pas que l'on instancie soi-même un tel comportement. Pourtant, la réponse usuelle à l'égard de cette considération semble être de caractère non conséquentialiste. Elle recommandera que les personnes cherchent à instancier de telles propriétés, même si cela devait avoir pour conséquence que moins de ces propriétés seront instanciées au total ; une telle approche est soutenue tant par les théories traditionnelles des obligations et des droits que par les versions plus récentes de la théorie contractuelle ${ }^{24}$.

Pour une fine argumentation à l'appui de cette interprétation, voir Sayre-McCord, G., « On Why Hume's 'General Point of View' Isn't Ideal — and Shouldn't Be », p. 202-228.

24. Sur la théorie des obligations et des droits, voir Dworkin, R., Taking Rights Seriously, London, Duckworth, 1978 (Prendre les droits au sérieux, trad. M.-J. Rossignol et 
Les concepts tributaires du sentiment, à l'opposé, sont de nature axiologique plutôt que déontologique. Ils se rapportent à la question qui vise à déterminer ce qui est le meilleur — ce qui est axios ou désirable —, laquelle s'applique, en principe, à presque toutes les classes de catégories conceptuelles : non seulement aux actions, par exemple, mais aussi aux personnes, aux mobiles et aux situations. L'une des approches axiologiques que de tels concepts peuvent contribuer à développer est celle de l'éthique de la vertu, laquelle se concentre sur les propriétés que des agents vertueux devraient instancier. Mais ce type d'approche peut aussi bien revêtir une forme de conséquentialisme, en dirigeant notre attention plutôt vers les propriétés que les individus devraient promouvoir, même si, parfois, les promouvoir ne veut pas dire qu'elles seront instanciées au niveau de leur propre comportement, dans leur psychologie ou encore dans le cadre de leurs relations avec autrui.

L'argumentation développée dans cet article suggère que les concepts tributaires de la conversation, essentiellement déontologiques, ont une certaine prééminence dans la pensée morale, puisqu'ils correspondent à ces considérations éthiques qui nous introduisent - et qui seules, peut-être, sont en mesure de nous introduire - dans l'espace des raisons éthiques. Mais cette suggestion demeure compatible, évidemment, avec la vue selon laquelle la question de savoir ce qu'il est juste ou mauvais de faire est déterminée, ultimement, par ce qui est le meilleur d'un point de vue général (autrement dit, par des considérations conséquentialistes). Je défends moi-même une telle conception, estimant que, aussi sérieuses que puissent être les exigences associées aux présupposés de la conversation — c'est-à-dire, aussi sérieuses que puissent être les exigences de civilité ou, comme nous pourrions aussi les qualifier, de courtoisie - elles devront être outrepassées dans des circonstances où il apparaît clair qu'il ne serait pas pour le mieux de les honorer ${ }^{25}$.

Je crois que le débat entre ces différentes approches de la théorie éthique, c'est-à-dire conséquentialisme et non conséquentialisme, devrait bénéficier de l'identification des évaluations de nature véritablement différente offertes à la pensée éthique par les concepts tributaires de la conversation et les concepts tributaires du sentiment. Il se peut qu'un échec à reconnaître cette divergence dans l'évaluation conceptuelle explique la polarisation des points de vue le long de cet axe, même si la reconnaissance de cette divergence n'argumente pas indiscutablement en faveur d'une quelconque position particulière. Une fois que nous constatons les voies divergentes par lesquelles notre espèce en arrive à conceptualiser les considérations éthiques,

F. Limare, Paris, PUF, 1995). Sur la question du contractualisme, voir Scanlon, T. M., What We Owe To Each Other, Cambridge (Mass.), Harvard University Press, 1998.

25. Voir Pettit, P., "The Consequentialist Perspective ", dans Baron, M., P. Pettit et M. Slote, Three Methods of Ethics : A Debate, Oxford, Blackwell, 1997. Voir aussi Pettit, P. et T. M. Scanlon, «Contractualism and Consequentialism », Theoria, 2000, p. 228-45. 
nous nous trouvons peut-être en meilleure posture pour évaluer les différentes positions éthiques auxquelles peut nous conduire une réflexion sur ces considérations $^{26}$.

26. J'ai pu bénéficié des échanges avec Allan Gibbard, Ossie Hanfling, Brad Hooker, Victoria McGeer, Susan Mendus, Michael Ridge, Michael Smith et Jay Wallace et des commentaires que m'ont fait parvenir les éditeurs de la revue Social Philosophy and Policy. Des conversations avec Stephen Darwall et Geoffrey Sayre-McCord m'ont particulièrement influencé et je conserve, à l'égard de chacun, une dette considérable. Cet article a également pu être amélioré, grâce aux discussions précieuses qui ont suivi sa présentation au Congrès du groupe de Philosophie sociale et Politique à La Jolla, Californie, en juin 2000 ; au Congrès Annuel de la Société britannique de Théorie éthique, tenu en Hollande, en juin 2000 ; et à un séminaire universitaire à l’Université du Nebraska, Lincoln, en septembre 2000. 